\title{
Estudo da eficiência do Orbignya oleifera como inibidor verde de corrosão para aço com baixo teor de carbono comparado com inibidor comercial em solução $\mathrm{HCl} 1 \mathrm{M}$
}

\author{
Study of the efficiency of Orbignya oleifera as a \\ green corrosion inhibitor for low carbon steel \\ compared to commercial inhibitor in \\ $1 \mathrm{M} \mathrm{HCl}$ solution
}

\author{
Jhonatan Peres ${ }^{1}$, Renato Conde ${ }^{2}$, Camilla Bezerra ${ }^{2}$, \\ Rita Costa ${ }^{2}$, Gedeon Reis ${ }^{1}$, Maria Eliziane Pires de Souza ${ }^{2}$, \\ Carmem Nascimento ${ }^{1}$
}

\footnotetext{
${ }^{1}$ Laboratório de Corrosão e Desgaste - IFMA/DMM/PPGEM, Campus Monte Castelo, CEP: 65030-005, São Luís, Maranhão, Brasil.

${ }^{2}$ Universidade Federal do Maranhão - UFMA, Campus São Luís Bacanga, CEP: 65065-545, São Luís, Maranhão, Brasil. e-mail: corrosaolab@gmail.com,jhonatan_ps.eng@live.com, renato-c.s@hotmail.com, bezerracamilla@hotmail.com, ritac.neta@hotmail.com, gedeonreis@ifma.edu.br, maria.eliziane@ufma.br, carmemfn@ifma.edu.br
}

\begin{abstract}
RESUMO
O trabalho visa um estudo comparativo entre a eficiência do óleo de coco babaçu (Orbignya oleifera) utilizado como inibidor verde de corrosão e um inibidor comercial testados em aço ABNT 1020 em meio contendo ácido clorídrico. A avaliação foi realizada utilizando técnicas eletroquímicas de polarização linear aliado ao Método de Extrapolação de Tafel e Espectroscopia de Impedância Eletroquímica. As concentrações dos inibidores utilizadas foram 3, 4 e 5 g/L em solução contendo ácido clorídrico $(\mathrm{HCl}) 1 \mathrm{M}$. Os resultados de espectroscopia de impedância eletroquímica mostraram que o Orbignya oleifera atua como inibidor de adsorção e que sua eficiência de inibição $(56,45 \%)$ é superior comparado a eficiência do inibidor comercial $(36,08 \%)$ na concentração de $3 \mathrm{~g} / \mathrm{L}$, uma vez que apresentou maior resistência de transferência de carga $(47,3 \Omega)$ em comparação ao inibidor HPAA $(32,23 \Omega)$. Os resultados de polarização linear mostraram que o óleo de Orbignya oleífera aumentou a eficiência de inibição no aço ABNT 1020 em solução de $\mathrm{HCl} 1 \mathrm{M}$ nas concentração de 3 e $4 \mathrm{~g} / \mathrm{L}$. A máxima eficiência inibidora do Orbignya oleífera neste ensaio foi de $48 \%$, que pode estar associada a composição química desse óleo, na qual consta a presença do ácido láurico com concentração de 47\%, o que pode ter contribuído para uma camada de inibição mais eficiente.
\end{abstract}

Palavras chave: aço carbono, inibidor verde, corrosão, técnicas eletroquímicas.

\begin{abstract}
The aim of this work is to compare the efficiency of babassu coconut oil (Orbignya oleifera) used as a green corrosion inhibitor and a commercial inhibitor tested on ABNT 1020 steel in hydrochloric acid medium. The search evaluation was accomplished with electromechanical techniques of linear polarizations and Tafel Extrapolation Method altogether with electrochemical impedance spectroscopy. The concentrations of the inhibitors were 3, 4 and $5 \mathrm{~g} / \mathrm{L}$ in a solution of IM hydrochloric acid (HCI). The results of electrochemical impedance spectroscopy shows that Orbignya oleifera acts as an adsorption inhibitor and its inhibition efficiency value $(56.45 \%)$ is higher when compared to the level of efficiency of the commercial inhibitor (36.08\%) in the concentration of $3 \mathrm{~g} / \mathrm{L}$ once that presented higher charge transfer resistance $(47.3 \Omega)$ when compared to the HPAA (32.23 $\Omega$ ). The results of Linear Polarization Resistance shows Orbignya oleifera oil increased the inhibition efficiency in ABNT 1020 steel in $1 \mathrm{M} \mathrm{HCl}$ solution at 3 and $4 \mathrm{~g} / \mathrm{L}$ concentrations. The level maximum inhibitory efficiency of Orbignya oleifera in this test was $48 \%$. That percentage must be associated to chemical composition of that oil which contains lauric acid in a concentration of $47 \%$ - that probably contributed to a high efficiency of inhibition layer.
\end{abstract}

Keywords: carbon steel, green inhibitor, corrosion, electrochemical techniques. 


\section{INTRODUÇÃO}

Os processos de corrosão geram inúmeros prejuízos no setor industrial, principalmente na linha de produção de produtos [1]. Adutoras, plataformas de petróleo, estruturas metálicas, tubulações, pontes e componentes de máquinas sofrem constantemente com este processo de degradação causando falhas em equipamentos, parada nas linhas de produção e elevados custos de manutenção. Dentre as diversas maneiras de evitar ou minimizar esses danos, os inibidores de corrosão têm se destacado como uma das técnicas versáteis utilizadas pela indústria, uma vez que é caracterizado pelo baixo custo [2-5].

A aceitação desses inibidores na linha industrial é justificado pelas excelentes propriedades anticorrosivas. Sua utilização pode ser vista em unidades de refino, tubulações de sistemas de resfriamento, caldeiras e em processos de produção de petróleo e gás [6-10].

Entretanto, muitos inibidores mostram-se agressivos ao meio ambiente por apresentarem cromatos e fosfatos de alta toxidade, recebendo forte atenção de autoridades internacionais. Neste sentido, pesquisas têm sido desenvolvidas utilizando inibidores ambientalmente corretos chamados de inibidores naturais ou inibidores verdes [11]. Dentre esses inibidores, os produtos naturais como óleos essenciais e extratos de plantas podem ser empregados como inibidores verdes de corrosão [12-14].

Dentre as ligas metálicas, o aço carbono é o que apresenta uma combinação favorável de propriedades, tornando assim amplamente utilizado em aplicações da engenharia [15-19]. Devido sua versatilidade em aplicações, este material muitas vezes é utilizado em condições de exposição atmosférica, podendo ser facilmente encontrado em grandes estruturas como pontes, viadutos, tubulações em estações de tratamento de água e componentes de máquinas [20-25].

A susceptibilidade à corrosão desse aço é limitada pelas condições a que é exposto, não formando uma película protetora em ambientes agressivos, sendo sensível aos íons cloretos (Cl-), brometos (Br-) e iodetos (I-), acelerando assim o processo de corrosão [22-27]. A degradação deste material juntamente com os aspectos prejudiciais do uso de inibidores comerciais justificam esta pesquisa, no sentido de encontrar inibidores que sejam amigáveis ao meio ambiente e que apresentem eficiências compatíveis ou superiores quando comparados com os inibidores comerciais.

Neste contexto, este trabalho tem por objetivo apresentar um comparativo entre a eficiência do óleo de coco babaçu (Orbignya oleifera) aplicado como inibidor verde de corrosão e um inibidor comercial testados em aço ABNT 1020 em meio contendo ácido clorídrico ( $\mathrm{HCl} 1 \mathrm{M})$ através de técnicas eletroquímicas de Polarização Potenciodinâmica aliado ao Método de Extrapolação de Tafel e Espectroscopia de Impedância Eletroquímica.

\section{MATERIAIS E MÉTODOS}

\section{1 Óleo vegetal Orbignya Oleífera}

O óleo de coco babaçu foi extraído a partir dos frutos prensados a frio por meio de processamento industrial e inseridos no sistema em estudo na condição de recebido pelo fornecedor nas concentrações de 3,4 e $5 \mathrm{~g} / \mathrm{L}$ de solução $\mathrm{HCl} 1 \mathrm{M}$.

A composição em ácidos graxos e estrutura química deste óleo pode ser visualizada na Tabela 1 e Figura 1 , respectivamente.

Tabela 1: Composição em ácidos graxos do Orbignya oleífera [28,29].

\begin{tabular}{ll}
\hline Ácidos Graxos & Babaçu (\%) \\
\hline Ácido Caprílico (C 8:0) & $4-6$ \\
Ácido Caprico (C 10:0) & $6-7$ \\
Ácido Láurico (C12:0) & $40-47$ \\
Ácido Mirístico (C 14:0) & $14-18$ \\
Ácido Palmítico (C 16:0) & $6-9$ \\
Ácido Esteárico (C 18:0) & $3-5$ \\
Ácido Oléico (C 18:1) & $12-16$ \\
Ácido Linoléico (C 18:2) & $1-2$ \\
Ácido Araquídico (C 20:0) & $<1$ \\
\hline
\end{tabular}




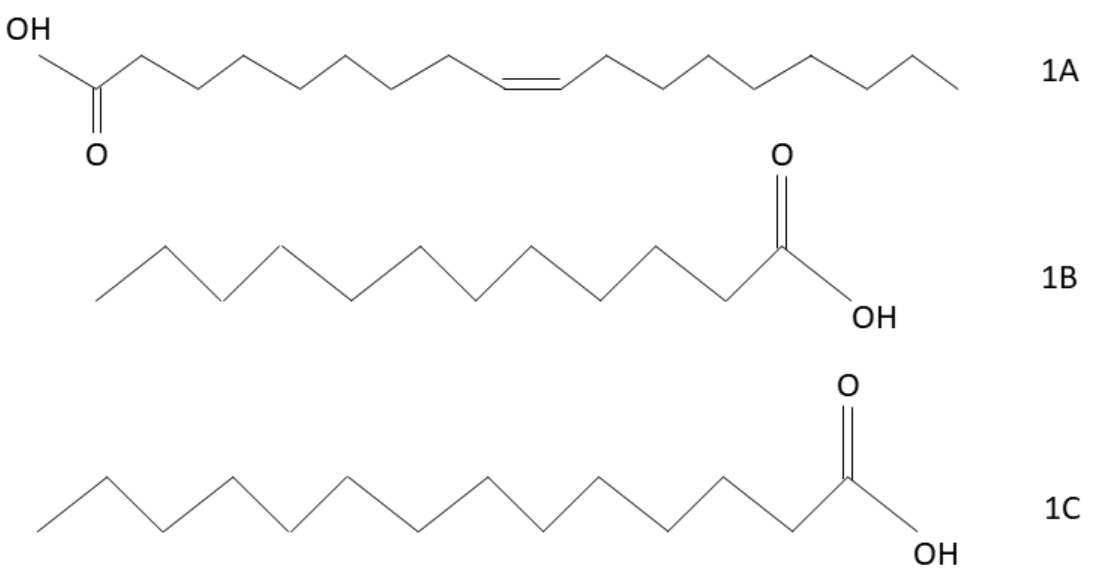

Figura 1: Principais constituintes do óleo vegetal. A) Ácido oleico (Ácido cis- $\Delta 9$-octadecenóico), B) Ácido laurico (Ácido dodecanóico) e C) Ácido mirístico (Ácido tetradecanóico) [28,29].

\subsection{Inibidor comercial HPAA}

O inibidor comercial utilizado foi o Polydisperse HPAA (Acido 2-Hidroxyfosfonocarboxílico). Segundo o fabricante, o produto é usado principalmente como inibidor de corrosão em sistema de água de recarga do campo petrolífero, em galvanoplastia (aço e ferro), setor petroquímico, usina produtora de energia e indústrias médicas. Está presente em instalações de tratamento de água industrial, torres de resfriamento e caldeiras, sendo aplicado como sequestrante de íons metálicos e inibidor de corrosão catódica [30]. As propriedades físico-químicas deste inibidor comercial estão apresentadas na Tabela 2

Tabela 2: Propriedades físico-químicas do HPAA (Acido 2-Hidroxyfosfonocarboxílico) [30]

\begin{tabular}{ll}
\hline Propriedades físico-químicas & \\
\hline Formula Molecular & $\mathrm{C}_{2} \mathrm{H}_{5} \mathrm{O}_{6} \mathrm{P}$ \\
Peso molecular & $156,03 \mathrm{~g} / \mathrm{mol}$ \\
Aparência & Líquido escuro \\
Concentração de sólidos & $50,0 \mathrm{mín}$ \\
Densidade $\left(20^{\circ} \mathrm{C}\right)$ & $1,3 \mathrm{~g} / \mathrm{cm}^{3}$ no mín. \\
pH Solução $1 \%$ & $1,0-3,0$ \\
\hline
\end{tabular}

\subsection{Amostras de aço carbono}

As amostras usadas como eletrodos foram de aço-carbono ABNT 1020, cuja composição pode ser visualizada na Tabela 3, a partir do qual foi confeccionado os eletrodos de trabalho com área superficial de $1 \mathrm{~cm}^{2}$, sendo embutidas a frio e lixadas com lixas d'água de granulometria variando de 200 a 1200 mesh. Em seguida foram lavadas com água e secas com jato de ar quente.

Tabela 3: Composição do aço ABNT 1020

\begin{tabular}{cccccc} 
Material & $\mathrm{C} \%$ & $\mathrm{Mn} \%$ & $\mathrm{Si} \%$ & $\mathrm{P} \%$ (Máx.) & $\mathrm{S} \%$ (Máx.) \\
\hline ABNT 1020 & 0,18 & 0,30 & 0,05 & 0,04 & 0,05 \\
\hline
\end{tabular}

\subsection{Ensaios eletroquímicos}

As medidas eletroquímicas foram realizadas em um potenciostato/galvanostato modelo PGSTAT 302N, controlado pelo software Nova (versão 1.11) da Metrohm Autolab B.V.

A montagem da célula eletroquímica foi composta por um eletrodo de trabalho (ET), um contra eletrodo (CE) e um eletrodo de referência (ER) $\mathrm{Ag} / \mathrm{AgCl}$ ( $\mathrm{KCl}$ saturado). Inicialmente foram realizadas medidas de potencial de circuito aberto (OCP) para garantir a estabilização do sistema, procedimento que atingiu $1800 \mathrm{~s}$.

Para os ensaios de polarização potenciodinâmica, a taxa de varredura aplicada foi de $1 \mathrm{mV} \cdot \mathrm{s}^{-1} \mathrm{com}$ sobretensões anódicas e catódicas de +300 e $-300 \mathrm{mV}$, respectivamente, em relação ao potencial de circuito 
aberto. A medição eletroquímica foi de 30 minutos. Para conhecimento dos resultados numéricos do ensaio foi aplicado o Método de Extrapolação de Tafel.

Nos ensaios de impedância, a frequiência aplicada variou de $10^{5}$ a $0,01 \mathrm{~Hz}$ com amplitude de pertubação de $10 \mathrm{mV}$. A montagem do circuito equivalente foi gerado no próprio software que faz portabilidade com o equipamento (Nova $1.1^{\circledR}$ ) e em seguida, os valores obtidos nos experimentos foram transportados para o software Origin ${ }^{\circledR}$ para serem tratados estatisticamente e analisados. Todos os experimentos foram realizados em triplicata para maior confiabilidade dos resultados e realizados a temperatura ambiente $\left(30^{\circ} \mathrm{C} \pm 1^{\circ} \mathrm{C}\right) \mathrm{sob}$ condições aeradas e não agitadas.

\section{RESULTADOS E DISCUSSÃO}

\subsection{Medidas de Polarização Linear}

O método de Extrapolação de Tafel aplicado para analisar a eficiência dos óleos como inibidores de corrosão segue a metodologia apresentada por Poorqasemi [31].

A Tabela 4 e Figura 2 mostram os resultados experimentais do ensaio de polarização potenciodinâmica para as concentrações em estudo. Observa-se que a presença do óleo de babaçu leva a um pequeno deslocamento do potencial para valores mais catódicos o que pode está relacionado a redução dos íons hidrogênio $\left(\mathrm{H}_{2(\mathrm{~g})}\right)$, de acordo com a reação da Equação 1, bem como uma diminuição nos valores de densidade de corrente de corrosão, quando comparados ao resultado sem o inibidor [32].

$$
2 H_{(a q)}^{+}+2 e^{-} \Rightarrow H_{2(g)}
$$

Tabela 4: Parâmetros eletroquímicos obtidos nos ensaios de polarização na presença do óleo de Orbignya oleífera

\begin{tabular}{llllll}
\hline $\mathbf{C}(\mathbf{g} / \mathbf{L})$ & Ecorr $(\mathbf{m V})$ & jcorr $\left(\boldsymbol{\mu A} / \mathbf{c m}^{2}\right)$ & $\begin{array}{l}-\boldsymbol{\beta c a t o ́ d i c o} \\
(\mathbf{m V / d e c})\end{array}$ & $\begin{array}{l}\boldsymbol{\beta} \\
(\mathbf{m V} / \mathbf{d e c})\end{array}$ & $\boldsymbol{\eta} \%$ \\
\hline Neutra & $-431,55$ & 700,84 & 70,99 & 103,05 & - \\
3 & $-437,72$ & 367,21 & 71,63 & 104,16 & $48 \%$ \\
4 & $-434,91$ & 448,15 & 71,19 & 96,85 & $36 \%$ \\
5 & $-436,54$ & 769,32 & 74,11 & 100,44 & Ineficiente \\
\hline
\end{tabular}

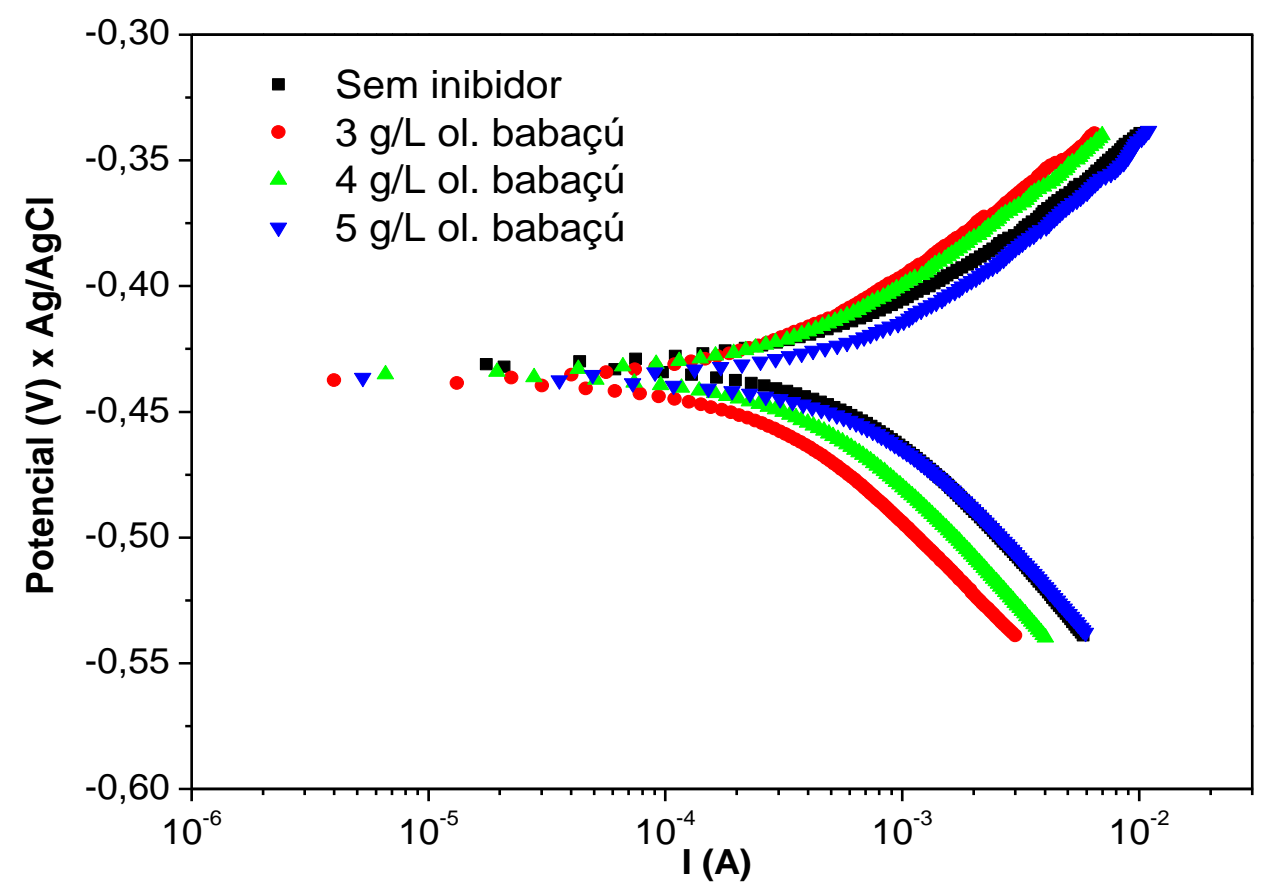

Figura 2: Curvas de polarização representativas de Tafel na ausência e presença de diferentes concentrações de Orbignya oleífera. 
A densidade de corrente no sistema neutro equivale a $700,84 \mu \mathrm{A} / \mathrm{cm}^{2}$ e a adição de $3 \mathrm{~g} / \mathrm{L}$ do óleo vegetal Orbignya oleífera reduz esse valor a $367,21 \mu \mathrm{A} / \mathrm{cm}^{2}$ atingindo a eficiência de $48 \%$ para este ensaio. A ação inibidora desse óleo é evidente na concentração de $3 \mathrm{~g} / \mathrm{L}$ com a diminuição da densidade de corrente.

As alterações de $\beta_{a}$ e $-\beta_{c}$ foram atribuidas por Torres [32] ao fato de que tanto as reações catódicas de evolução do hidrogênio com as reações anódicas de dissolução metálica foram inibidas principalmente pela redução da área ativa em virtude da formação de uma monocamada de moléculas do óleo adsorvida na superfície do aço carbono, diminuindo o processo de corrosão.

Os testes realizados na concentração de $4 \mathrm{~g} / \mathrm{L}$ do óleo demonstraram uma redução na densidade de corrente para $448,15 \mu \mathrm{A} / \mathrm{cm}^{2}$ ocasionando uma menor eficiência (36\%). A eficiência encontrada nas concentrações de 3 e $4 \mathrm{~g} / \mathrm{L}$ pode estar associada a composição química desse óleo, na qual consta a presença do ácido láurico com concentração de $47 \%$ no óleo de babaçu, o que pode ter contribuído para uma camada de inibição mais eficiente. Estudo semelhante foi encontrado por Machado [26], além da ação do ácido oléico, presente neste óleo na concentração de 12 a 16\%, como mostra as pesquisas realizadas por Alconchel-Gago [33].

Por outro lado, os testes realizados na concentração de $5 \mathrm{~g} / \mathrm{L}$ indicam que existe uma quantidade específica para atuação do óleo como inibidor, apresentando densidade de corrente maior que o sistema na ausência do inibidor $\left(769,32 \mu \mathrm{A} / \mathrm{cm}^{2}\right)$, sendo ineficiente nesta proporção.

A partir dos valores das densidades de corrente obtidas nos experimentos, a eficiência de inibição $\eta \%$ foi calculada conforme a Equação 2:

$$
\eta \%=\frac{j_{s i}-j_{c i}}{j_{s i}} \times 100
$$

onde $j_{s i}$ é a densidade de corrente na ausência do inibidor e $j_{c i}$ é densidade de corrente na presença do inibidor.

Os resultados de polarização potenciodinâmica com o uso do inibidor comercial HPAA em variadas concentrações é apresentado na Tabela 5 e Figura 3.

Tabela 5: Parâmetros eletroquímicos obtidos na presença de diferentes concentrações de HPAA (Acido 2Hidroxyfosfonocarboxílico).

\begin{tabular}{llllll}
\hline $\mathbf{C}(\mathbf{g} / \mathbf{L})$ & $\mathbf{E c o r r}(\mathbf{m V})$ & $\mathbf{J c o r r}\left(\boldsymbol{\mu \mathbf { A } / \mathbf { c m } ^ { 2 } )}\right.$ & $\begin{array}{l}\boldsymbol{\beta c} \\
\mathbf{m V} / \mathbf{d e c})\end{array}$ & $\begin{array}{l}\boldsymbol{\beta a} \\
(\mathbf{m V} / \mathbf{d e c})\end{array}$ & $\boldsymbol{\eta} \%$ \\
\hline Neutra & $-431,55$ & 700,84 & 70,99 & 103,05 & - \\
3 & -440 & 328,33 & 67,91 & 94,41 & $53 \%$ \\
4 & $-436,95$ & 294,69 & 66,76 & 98,73 & $58 \%$ \\
5 & -444 & 278,85 & 71,95 & 101,06 & $60 \%$ \\
\hline
\end{tabular}




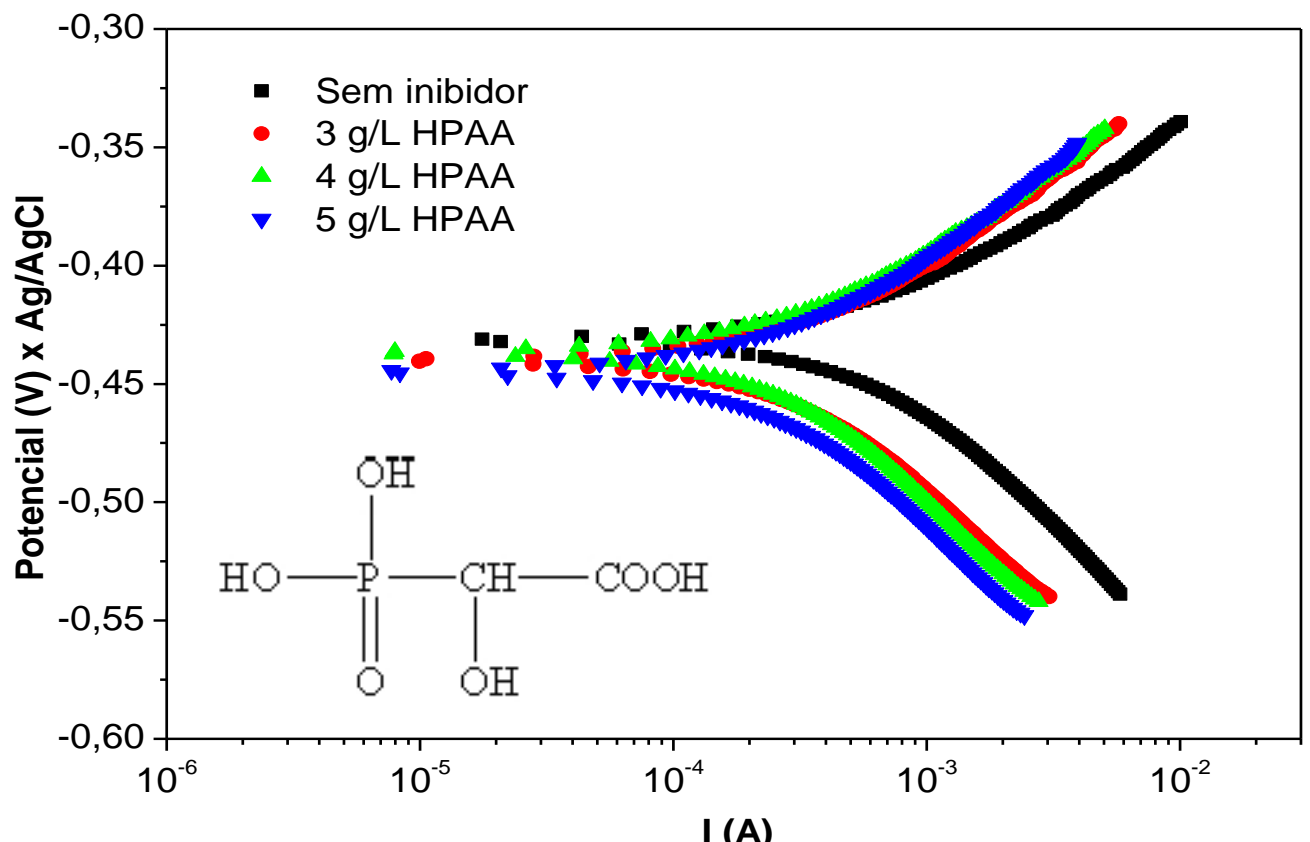

Figura 3: Curvas de polarização representativas de Tafel na presença de diferentes concentrações de HPAA (Acido 2Hidroxyfosfonocarboxílico).

Observa-se que a eficiência de corrosão aumentou com o aumento da concentração de inibidor, reduzindo os valores de densidade de corrente, alcançando assim a eficiência máxima de $60 \%$ na concentração de $5 \mathrm{~g} / \mathrm{L}$ de HPAA, ocorrendo predominância do efeito catódico nos ensaios, onde as reações catódicas são retardadas para menores valores de corrente. Segundo Gentil [34], os inibidores com efeito catódico são substâncias que liberam íons metálicos capazes de reagir com a alcalinidade catódica, produzindo precipitados insolúveis que atuam restringindo as reações catódicas. As regiões catódicas são cobertas por este composto insolúvel dificultando assim a difusão do oxigênio e a condução de elétrons, que por sua vez inibe o processo catódico. Essa inibição promove uma acentuada polarização catódica.

Ainda neste contexto, a eficiência do inibidor HPAA pode estar relacionada a presença de $\mathrm{P}$ na sua estrutura química, uma vez que sua presença garante a função polar da estrutura. De acordo com Yaro [13], a função polar tem grande influência na reação para o estabelecimento do processo de adsorção. Como abordado por alguns pesquisadores [14,19], as ligações $\sigma$ e $\pi$ presentes na estrutura são também extremamente eficientes e possibilitam a ocorrência deste processo.

\subsection{Medidas de Impedância Eletroquímica}

Para análise das curvas de impedância é comum correlacionar os dados com um circuito elétrico equivalente onde a variação dos valores dos componentes do circuito representam os processos físicos e químicos que ocorrem no sistema investigado [34,35]. Os espectros de impedância eletroquímica ou diagrama de "Nyquist" são representados em termos de Z' e Z", sendo parte real e parte imaginária no plano complexo respectivamente.

A Tabela 6 e Figura 4 mostram os resultados obtidos experimentalmente e as curvas em diferentes concentrações de Orbignya oleífera respectivamente.

Os valores de Cdl podem ser calculados com base na Equação 3:

$$
C_{\mathrm{dl}}=\left(\mathrm{Y}_{0} \cdot \mathrm{R}_{\mathrm{p}}^{1-\mathrm{n}}\right)^{\frac{1}{\mathrm{n}}}
$$

onde $Y_{0}$ representa os valores do CPE $(\mathrm{S}), \mathrm{R}_{\mathrm{p}}$ a resistência de transferência de carga $(\Omega)$ e $\mathrm{n}$ representa o índice que mede a perfeição do elemento CPE, podendo este varia de 0 a 1 . Dependendo do valor de $n$, CPE pode representar resistência $\left(n=0, \mathrm{Y}_{0}=1 / \mathrm{R}\right)$, capacitância $\left(n=1, \mathrm{Y}_{0}=\mathrm{C}\right)$, indutância $\left(n=1, \mathrm{Y}_{0}=\right.$ $1 / \mathrm{L})$ ou impedância de Warburg $\left(n=0.5, \mathrm{Y}_{0}=1 / \sigma \sqrt{2}\right)$. 
Os valores de eficiência $n \%$ na Tabela 6 podem ser calculados a partir dos valores de Rp para as concentraçoes estudadas através da Equação 4:

$$
\eta \%=\frac{R_{p}-R_{p, o}}{R_{p}} \times 100
$$

onde $R_{p}$ e $R_{p, o}$ representam as resistências de transferências de carga na presença e na ausência do óleo em estudo respectivamente.

Tabela 6: Parâmetros eletroquímicos obtidos por Nyquist na ausência e presença de variadas concentrações de Orbignya oleífera em solução $\mathrm{HCl} 1 \mathrm{M}$.

\begin{tabular}{|c|c|c|c|c|c|c|}
\hline \multirow{2}{*}{$C(g / L)$} & \multirow{2}{*}{ Rs ( $(\mathbf{Q})$} & \multirow{2}{*}{$\operatorname{Rp}\left(\Omega . \mathrm{cm}^{-2}\right)$} & \multicolumn{3}{|l|}{ CPE } & \multirow{2}{*}{$\boldsymbol{\eta} \%$} \\
\hline & & & $Y_{0}(\mu S)$ & $\operatorname{Cdl}\left(\mu \mathrm{F} . \mathrm{cm}^{-2}\right)$ & $n$ & \\
\hline Neutra & 1,59 & 20,6 & 275 & 124 & 0,86 & - \\
\hline 3 & 1,74 & 47,3 & 221 & 110 & 0,86 & $56,45 \%$ \\
\hline 4 & 1,86 & 35,5 & 282 & 135 & 0,86 & $41,97 \%$ \\
\hline 5 & 1,56 & 18,2 & 266 & 107 & 0,85 & Ineficiente \\
\hline
\end{tabular}

Nota: Rs representa a resistência da solução, $R p$ a resistência de transferência de carga, Yo representa os valores do elemento de fase constante (CPE), Cdl a capacitância da dupla camada elétrica e n presenta o índice que mede a perfeição desse elemento.

O gráfico de Nyquist permite observar a variação em termos de resistência do sistema num dado intervalo de frequência de interesse. Graficamente, quanto maior o arco apresentado no espectro, maior a resistência à transferência de cargas, ou passagem de corrente. Em altas frequências, a resistência apresentada é a resistência do eletrólito e a baixas frequências, o somatório das resistências ( $\mathrm{Rs}+\mathrm{Rp})$ dominam o sistema.

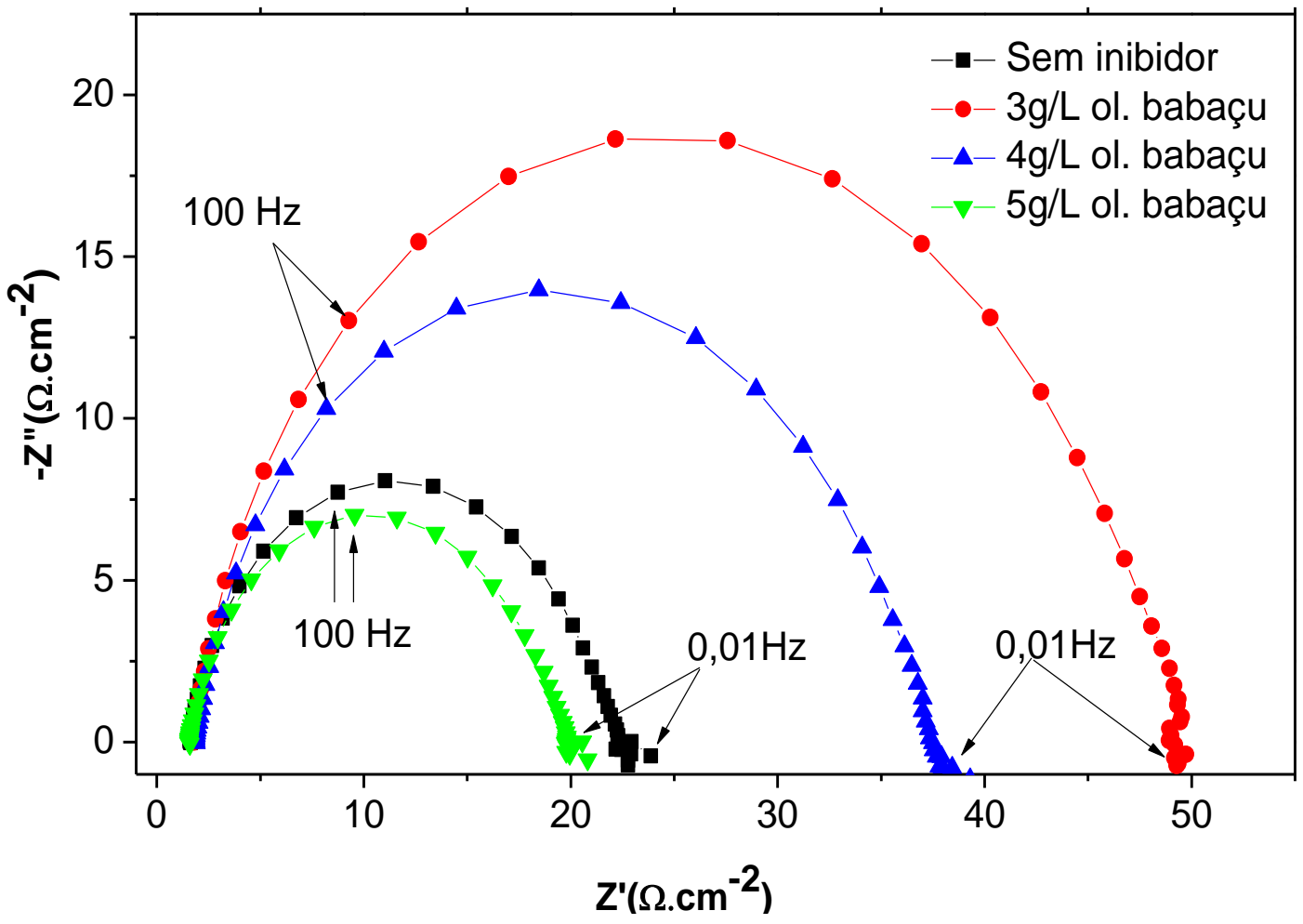

Figura 4: Espectros de Nyquist na ausência e presença de variadas concentrações de Orbignya oleífera em solução $\mathrm{HCl}$ $1 \mathrm{M}$. 
Nesse sentido, observa-se que a presença do óleo de babaçu nas concentrações de 3 e $4 \mathrm{~g} / \mathrm{L}$, induz a uma elevação no sistema de inibição, uma vez que os valores de Rp são elevados para 47,3 e 35,5 2 . Observa-se também que a solução se torna mais resistiva nestas concentrações, pois os novos valores de Rs são maiores comparados ao sistema na ausência do óleo. Os valores representativos de Cdl são também alterados, fenômeno atribuído a uma modificação da estrutura na dupla camada elétrica, bem como uma alteração na constante dielétrica [36].

A concentração de $5 \mathrm{~g} / \mathrm{L}$ não gera um resultado satisfatório, o que leva a supor que há uma concentração limitante para que o efeito de inibição seja satisfatório. A eficiência máxima obtida com este óleo foi $56,45 \%$ na presença de $3 \mathrm{~g} / \mathrm{L}$ de óleo de babaçu.

A análise dos dados foi realizada a partir do circuito equivalente da Figura 5. O circuito apresenta capacitores e resistores devido a ocorrência dos fenômenos atuantes de interação eletrônica. Para o inibidor HPAA, os resultados de EIE (Espectroscopia de Impedância Eletroquímica) também são apresentados em forma de Nyquist.

A $R_{p}$ foi calculada pelo somatório das resistências obtidas em cada arco capacitivo $\left(R_{p}=R_{p 1}+R_{p 2}\right)$ [37]. Como pode ser observado na Figura 6, é nítida a influência do inibidor nos resultados de impedância.

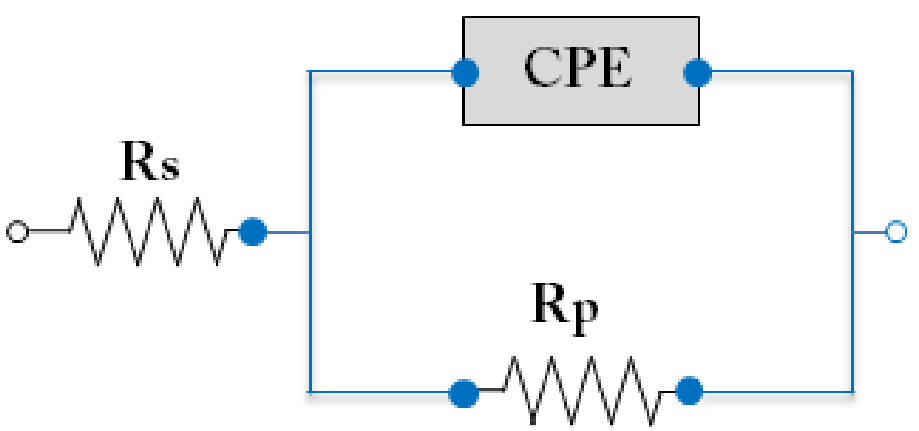

Figure 5: Circuito equivalente $[\mathrm{R}(\mathrm{RQ})]$ gerado a partir do ajuste dos dados experimentais para Orbignya oleífera em solução $\mathrm{HCl} 1 \mathrm{M}$.

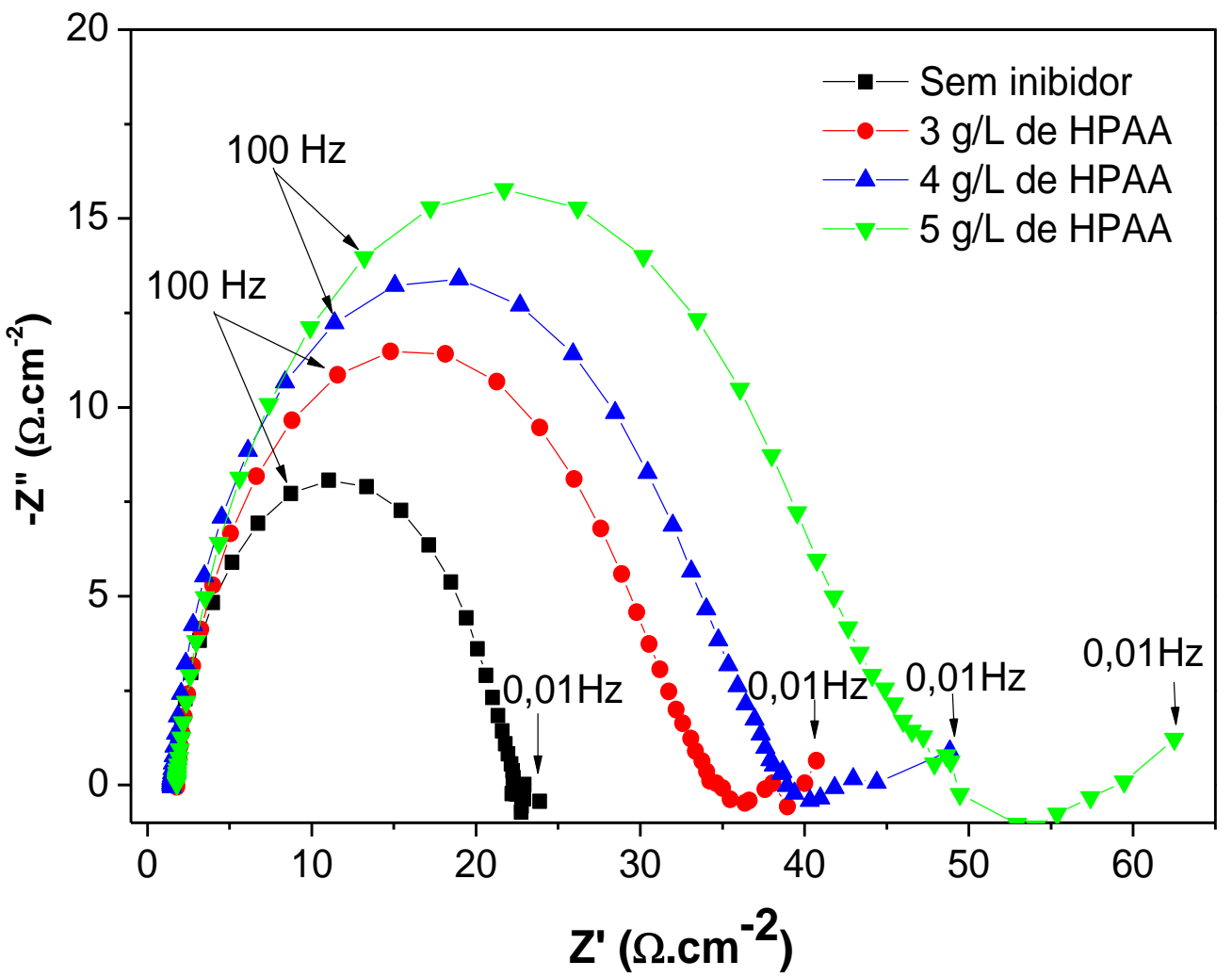

Figura 6: Espectros de Nyquist na ausência e presença de variadas concentrações de HPAA em solução HCl 1M. 
Na Tabela 7 estão tabulados os principais resultados do ensaio. Observa-se um aumento da resistência de transferência de carga mediante maiores concentrações do inibidor, uma vez que proporciona a formação de maiores arcos capacitivos em comparação ao sistema na ausência do inibidor. A Rp obtida com este inibidor na concentração de $5 \mathrm{~g} / \mathrm{L}$ foi de $45,69 \Omega$ que em comparação ao sistema em branco $(20,6 \Omega)$ resulta numa

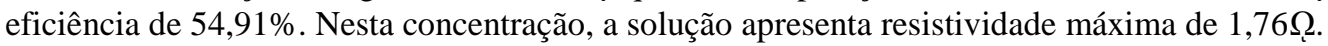

Observa-se também, de maneira geral, uma diminuição nos valores de capacitância com o aumento da concentração de inibidor. Este fenômeno foi atribuído por Pradityana [36] a uma diminuição da constante dielétrica ou até mesmo um aumento da densidade da dupla camada elétrica como resultado da adsorção de moléculas na interface metal/solução.

Tabela 7: Parâmetros obtidos por Nyquist na ausência e presença de variadas concentrações de HPAA em solução $\mathrm{HCl}$ $1 \mathrm{M}$

\begin{tabular}{|c|c|c|c|c|c|c|c|c|c|c|c|}
\hline \multirow[b]{2}{*}{$C(g / L)$} & \multirow[b]{2}{*}{$\operatorname{Rs}(\Omega)$} & \multirow{2}{*}{$\begin{array}{l}\mathbf{R}_{\mathrm{p} 1} \\
\left(\Omega_{\mathrm{.} . \mathrm{cm}^{-2}}\right)\end{array}$} & \multicolumn{3}{|l|}{ CPE1 } & \multirow{2}{*}{$\begin{array}{l}\mathbf{R}_{\mathrm{p} 2} \\
\left(\Omega_{.} . \mathrm{cm}^{-2}\right)\end{array}$} & \multicolumn{3}{|l|}{ CPE2 } & \multirow{2}{*}{$\begin{array}{l}R_{p} \\
\left(\Omega . c^{-2}\right)\end{array}$} & \multirow[b]{2}{*}{$\boldsymbol{\eta} \%$} \\
\hline & & & $\mathbf{Y}_{0}(\boldsymbol{\mu S})$ & $\begin{array}{l}\text { Cdl } \\
\left(\mu \mathrm{F} . \mathrm{cm}^{-2}\right)\end{array}$ & $n$ & & $\begin{array}{l}\mathbf{Y}_{0} \\
(\mathbf{m S})\end{array}$ & $\begin{array}{l}\text { Cdl } \\
\left(\mathrm{mF} . \mathrm{cm}^{-2}\right)\end{array}$ & $n$ & & \\
\hline Neutra & 1,59 & 20,6 & 275 & 124 & 0,866 & - & - & - & - & 20,6 & - \\
\hline 3 & 1,76 & 27,4 & 180 & 88 & 0,881 & 4,83 & 15,99 & 8,04 & 0,788 & 32,23 & $36,08 \%$ \\
\hline 4 & 1,45 & 30,65 & 156 & 86 & 0,899 & 5,93 & 12,99 & 6,35 & 0,781 & 36,58 & $43,69 \%$ \\
\hline 5 & 1,76 & 38,09 & 163 & 77 & 0,871 & 7,6 & 18,84 & 9,42 & 0,737 & 45,69 & $54,91 \%$ \\
\hline
\end{tabular}

Os valores de frequência revelam a particularidade de cada sistema. Embora todos os experimentos tenham sido realizados na mesma faixa de frequência $\left(10^{5} \mathrm{~Hz}-10^{-2} \mathrm{~Hz}\right)$, observa-se diferentes valores de impedância para cada concentração em estudo.

Os ensaios com Orbignya oleífera mostraram que o óleo apresenta uma boa eficiência de inibição em algumas concentrações. Por outro lado, o aumento na concentração do inibidor comercial levou a um aumento nessa eficiência de inibição, apresentando um arco capacitivo maior em todos os testes com HPAA.

A Figura 9 ilustra o circuito equivalente que melhor representa os dados experimentais para este teste.

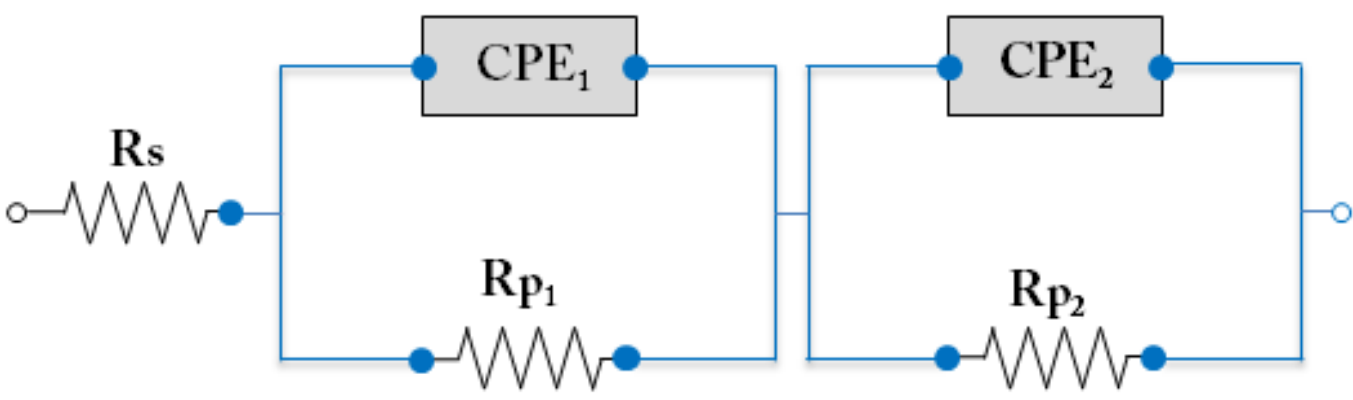

Figure 7: Circuito equivalente $[\mathrm{R}(\mathrm{RQ})(\mathrm{RQ})]$ que melhor se adequa aos dados experimentais para HPAA em solução $\mathrm{HCl}$ $1 \mathrm{M}$.

\subsection{Resultados comparativos}

Como foi observado, o óleo de babaçu na concentração de $3 \mathrm{~g} / \mathrm{L}$ foi a que gerou melhor desempenho, sendo assim, os resultados para essa concentração serão expostos de forma comparativa, a fim de melhorar a visualização do comportamento entre os inibidores.

A Figura 8 e 9 apresenta os diagramas de Nyquist e as curvas de polarização comparativas para o $O r$ bignya oleífera e o HPAA, ambos a 3g/L em solução $\mathrm{HCl} 1 \mathrm{M}$. Nesta ilustração, os valores de Rs e Rp são expressos nos espectros de impedância a fim de facilitar a análise comparativa.

Através do comportamento das curvas nesta concentração, é possível notar que a atuação do óleo de babaçu é semelhante ao inibidor comercial, deslocando o potencial de corrosão para região mais catódica e diminuindo a corrente de corrosão. 
Observa-se, graficamente, que as curvas estão praticamente sobrepostas, o que leva a entender que nessa concentração o efeito da presença do óleo de babaçu é similar ao comercial. Através dos dados das Tabelas 4 e 5, nota-se a diferença entre as inclinações catódicas e anódicas.

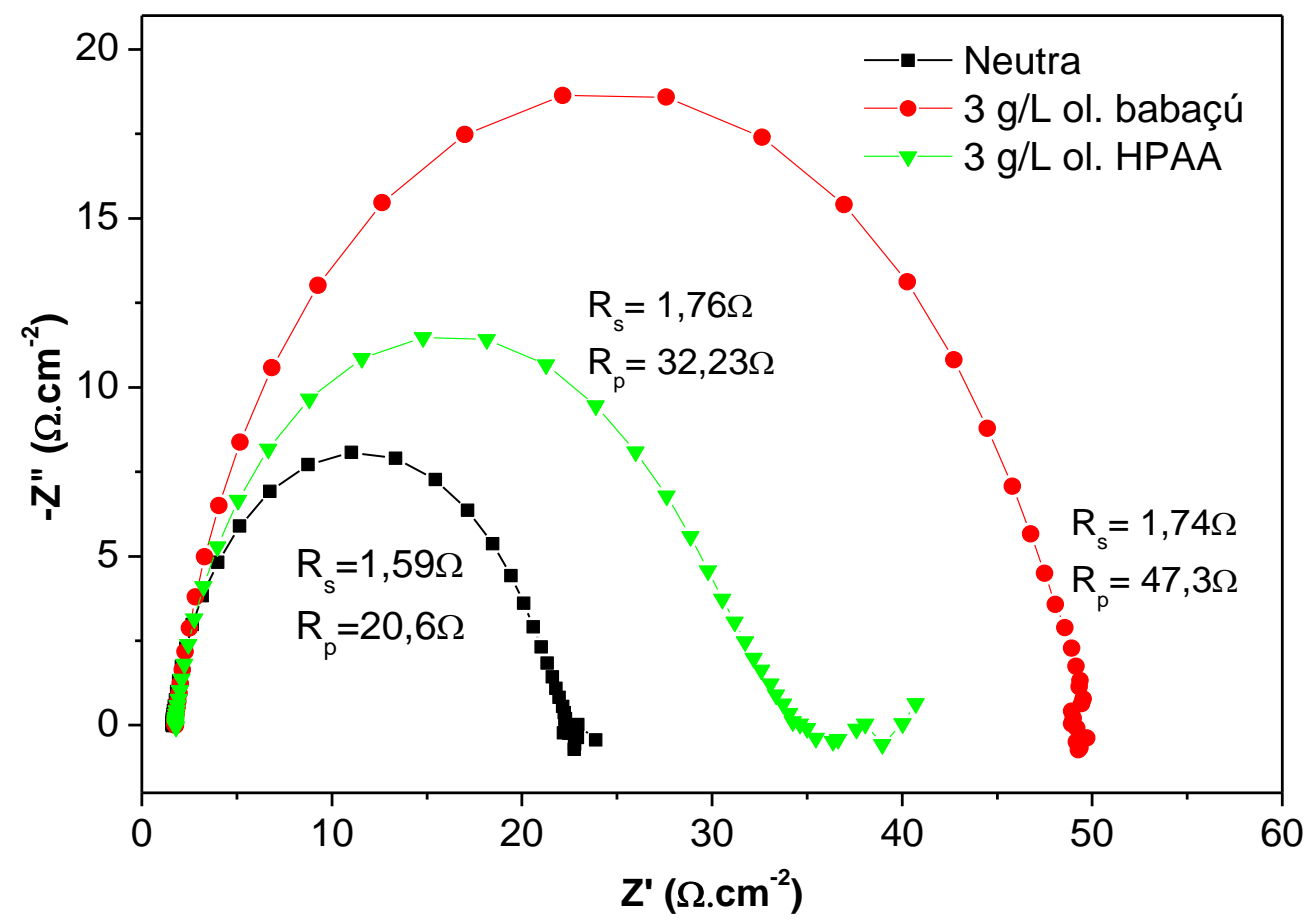

Figura 8: Comparativo do diagrama de Nyquist do Orbignya oleífera e HPAA a 3g/L em solução HCl 1M.

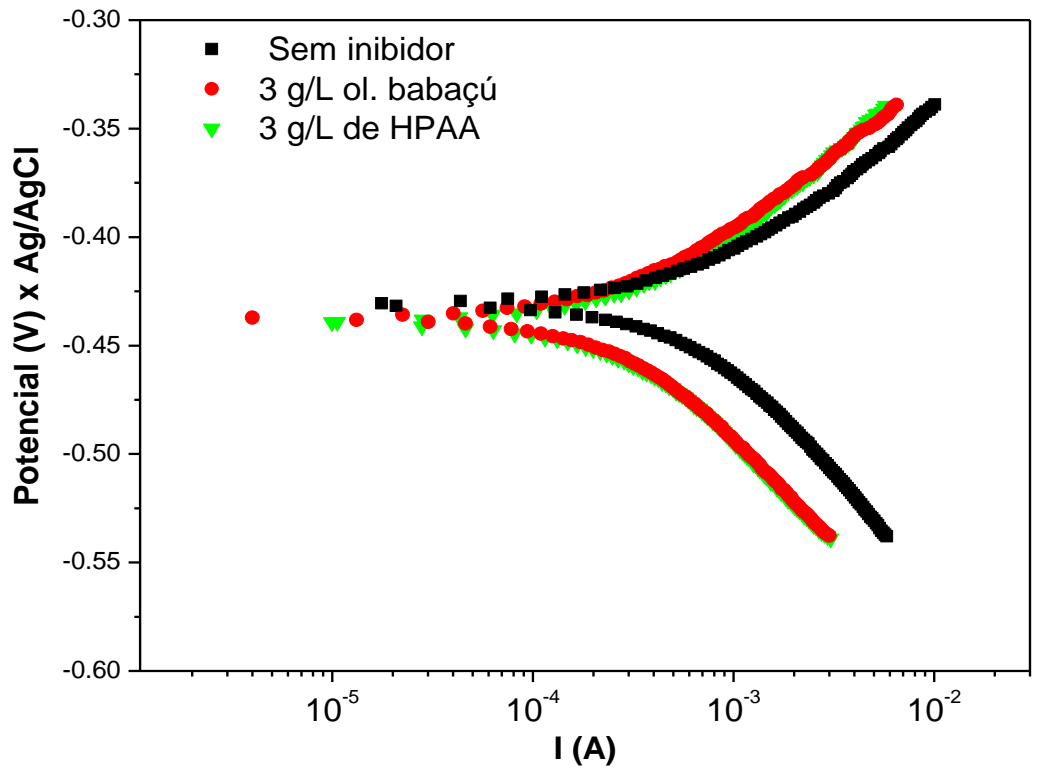

Figura 9: Comparativo do diagrama de Polarização entre o Orbignya oleífera e HPAA a 3g/L em solução HCl 1M.

Para o óleo de babaçu, a $3 \mathrm{~g} / \mathrm{L},-\beta_{c}=71,63 \mathrm{mV} /$ dec e $\beta_{a}=104,16 \mathrm{mV} / \mathrm{dec}$, enquanto que para o inibidor HPAA na mesma concentração, $-\beta_{c}=67,91 \mathrm{mV} /$ dec e $\beta_{a}=94,41 \mathrm{mV} / \mathrm{dec}$. A proximidade desses valores pode estar associada a velocidades similares de corrosão em ambos os sistemas.

É possível observar que o óleo de babaçu é o que apresenta um melhor resultado, com $R_{p}=47,3 \Omega$, quando comparado ao inibidor comercial $\left(R_{p}=32,23 \Omega\right)$. Estes valores revelam que a presença do óleo de 
babaçu, nesta concentração, reduz a transferência eletrônica no processo de maneira mais efetiva que o inibidor HPAA.

\subsection{Mecanismo de inibição}

O efeito inibidor do Orbignya oleífera e HPAA observado no aço de baixo carbono em solução $\mathrm{HCl} 1 \mathrm{M}$ pode ser explicado pelo processo de adsorção dos mesmos na superfície do metal. Mediante os resultados de Polarização Potenciodinâmica e Espectroscopia de Impedância Eletroquímica obtidos neste trabalho é possível propor um mecanismo de ação dos compostos no combate a corrosão.

Baseado em Li et. al [38], as moléculas $\mathrm{H}_{2} \mathrm{O}$ presentes no eletrólito também poderiam se adsorver na interface metal/solução. Entretanto, a adsorção de compostos podem ocorrer por substituição entre o composto $[\mathrm{Org}(\mathrm{sol})]$ e moléculas de água na superfície metálica. Para o caso particular, sugere-se a reação da Equação 5 apresentada por Gerengi [39] como fundamental para compreensão do mecanismo descrito, uma vez que engloba a atuação mista dos compostos.

$$
\operatorname{Org}_{(\text {sol) }}+x \mathrm{H}_{2} \mathrm{O} \rightarrow \operatorname{Org}_{(\text {ads })}+x \mathrm{H}_{2} \mathrm{O}_{\text {(sol) }}
$$

onde x representa a relação entre o número de moléculas de $\mathrm{H}_{2} \mathrm{O}$ substituído pelo composto. Entendese que o primeiro passo para a adsorção de um inibidor geralmente envolve a substituição de uma ou mais moléculas de $\mathrm{H}_{2} \mathrm{O}$ adsorvidas na superfície do metal [39].

\section{CONCLUSÕES}

Os resultados das medidas eletroquímicas mostraram que o óleo de Orbignya oleífera aumentou a eficiência de inibição no aço ABNT 1020 em solução de HCl 1M nas concentração de 3 e 4 g/L. Nos ensaios de Polarização, a eficiência obtida com a aplicação de $3 \mathrm{~g} / \mathrm{L}$ de Orbignya oleífera foi de $48 \%$, valor este relativamente próximo a eficiência obtida com a mesma concentração de HPAA (53\%). Já nos ensaios de EIE, o Orbignya oleífera apresentou maior resistência de transferência de carga atingindo uma eficiência de 56,45\%, superando assim, a eficiência do inibidor HPAA $(36,08 \%)$ na concentração de 3 g/L. O óleo Orbignya oleífera em determinadas concentrações reduziu as densidades de corrente como efeito do processo de adsorção na superfície metálica atuando nas reações catódicas e anódicas. Uma vez que o mesmo se apresenta como não tóxico, barato, disponível na natureza em abundância e de fácil extração, pode-se assim sugerir continuidade em estudos para a aplicação deste óleo como inibidor verde de corrosão.

\section{AGRADECIMENTOS}

Ao PPGEM/Departamento de Mecânica e Materiais, IFMA - Campus Monte Castelo, São Luís - MA, Brasil, pelo fornecimento das instalações instrumentais de laboratório. A Fundação de Amparo à Pesquisa e ao Desenvolvimento Científico e Tecnológico do Maranhão - FAPEMA pelo apoio financeiro.

\section{BIBLIOGRAFIA}

[1] AL-OTAIBI, M.S., AL-MAYOUF, A.M., KHAN, M., et al., "Corrosion inhibitory action of some plant extracts on the corrosion of mild steel in acidic media", Arabian Journal of Chemistry, v. 7, n. 3, pp. 340346, 2014.

[2] OBOT, I.B., OBI-EGBEDI, N.O., UMOREN, S.A., "Antifungal drugs as corrosion inhibitors for aluminium in 0.1 M HCl”, Corrosion Science, v. 51, n. 8, pp. 1868-1875, 2009.

[3] DARIVA, C.G., GALIO, A.F., "Corrosion inhibitors-principles, mechanisms and applications", Developments in corrosion protection, IntechOpen, pp. 365-379, 2014.

[4] GARCIA, L. P., SANTOS, A.R., "Aplicação de inibidores de corrosão na indústria de Petróleo e Gás", In: $1^{\circ} E P G$, v. 2 , n. 1, pp. 33, 2013.

[5] NEGM, N.A., KANDILE, N.G., BADR, E.A., et al., "Gravimetric and electrochemical evaluation of environmentally friendly nonionic corrosion inhibitors for carbon steel in $1 \mathrm{M} \mathrm{HCl}$ ", Corrosion Science, v. 65, pp. 94-103, 2012. 
[6] ABDEL-GABER, A.M., ABD-EL-NABEY, B. A., KHAMIS, E., et al., "A natural extract as scale and corrosion inhibitor for steel surface in brine solution”, Desalination, v. 278, n. 1, pp. 337-342, 2011.

[7] BENSABAH, F., ESSAHLI, M., LAMIRI, A., et al., "Chemical Composition and Inhibitory Effect of the Essential Oil from Lippia Citriodora Irrigated by Wastewater on the Alkaline Corrosion of Aluminum", Portugaliae Electrochimica Acta, v. 32, n. 6, pp. 381-393, 2014.

[8] SINGH, A.K., MOHAPATRA, S., PANI, B., "Corrosion inhibition effect of Aloe Vera gel: Gravimetric and electrochemical study”, Journal of Industrial and Engineering Chemistry, v. 33, pp. 288-297, 2016.

[9] AFIA, L., SALGHI, R., BENALI, O., et al., "Electrochemical Evaluation of Linseed Oil as Environmentfriendly Inhibitor for Corrosion of Steel in $\mathrm{HCl}$ Solution”, Portugaliae Electrochimica Acta, v. 33, n. 3, pp. 137-152, 2015.

[10] MIGAHED, M.A., ATTYA, M.M., RASHWAN, S.M., et al., "Synthesis of some novel non ionic surfactants based on tolyltriazole and evaluation their performance as corrosion inhibitors for carbon steel", Egyptian Journal of Petroleum, v. 22, n. 1, pp. 149-160, 2013.

[11] HEGAZY, M.A., EL-ETRE, A.Y., EL-SHAFAIE, M., et al., "Novel cationic surfactants for corrosion inhibition of carbon steel pipelines in oil and gas wells applications", Journal of Molecular Liquids, v. 214, pp. 347- 356, 2016.

[12] HEAKAL, F.E., ELKHOLY, A.E., "Gemini Surfactants as Corrosion Inhibitors for Carbon Steel”, Journal of Molecular Liquids, v. 230, pp. 395-407, 2017.

[13] YARO, A.S., KHADOM, A.A., WAEL, R.K., et al., "Apricot juice as green corrosion inhibitor of mild steel in phosphoric acid”, Alexandria Engineering Journal, v. 52, n. 1, pp. 129-135, 2013.

[14] SANYAL, B., "Organic compounds as corrosion nhibitors in different environments - a review", Progress in Organic Coatings, v. 9, n. 2, pp. 165-236, 1981.

[15] GUO, L., OBOT, I.B., ZHENG, X., et al.,"Theoretical insight into an rule about organic corrosion inhibitors containing nitrogen, oxygen, and sulfur atoms”, Applied Surface Science, v. 406, pp. 301-306, 2017.

[16] EL-HADDAD, M.N., "Chitosan as a green inhibitor for copper corrosion in acidic médium”, International Journal of Biological Macromolecules, v. 55, pp. 142-149, 2013.

[17] GAYATHRI, V.S., YAMUNA, K., GNANA P.D., et al., "Green inhibitors: Anti corrosive propensity of Garcinia mangostana for Aluminum 1100", Solid State Phenomena, Trans Tech Publications, v. 185, pp. 109-112, 2012.

[18] BOUMHARA, K., TABYAOUI, M., JAMA, C., et al., “Artemisia Mesatlantica essential oil as green inhibitor for carbon steel corrosion in $1 \mathrm{M} \mathrm{HCl}$ solution: Electrochemical and XPS investigations", Journal of Industrial and Engineering Chemistry, v. 29, pp. 146-155, 2015.

[19] EL-ETRE, A.Y., ALI, A.I., "A novel green inhibitor for C-steel corrosion in 2.0 mol.L ${ }^{-1}$ hydrochloric acid solution”, Chinese Journal of Chemical Engineering, v. 25, n. 3, pp. 373-380, 2017.

[20] JOKAR, M., FARAHANI, T.S., RAMEZANZADEH, B., "Electrochemical and surface characterizations of morus alba pendula leaves extract (MAPLE) as a green corrosion inhibitor for steel in $1 \mathrm{M} \mathrm{HCl}$ ", Journal of the Taiwan Institute of Chemical Engineers, v. 63, pp. 436-452, 2016.

[21] FATTAH-ALHOSSEINI, A., NOORI, M., "Corrosion inhibition of SAE 1018 carbon steel in $\mathrm{H}_{2} \mathrm{~S}$ and $\mathrm{HCl}$ solutions by lemon verbena leaves extract", Measurement, v. 94, pp. 787-793, 2016.

[22] SANTOS, J.E.P.D., BARROSO, A.P.R.N., PARENTE, M.M.V., "Study of the Corrosion Inhibition of Carbon Steel in acidic chloride by Mangiferin”, Matéria (Rio de Janeiro), v. 21, n. 4, pp. 1045-1053, 2016.

[23] MOBIN, M., RIZVI, M., "Polysaccharide from Plantago as a green corrosion inhibitor for carbon steel in 1M HCl solution”, Carbohydrate Polymers, v. 160, pp. 172-183, 2017.

[24] CURVELO, F.M., "Uma imersão no tabuleiro da baiana: o estudo do óleo de palma”, Dissertação de M.Sc., Universidade Federal da Bahia, Brasil, 2010.

[25] OLIVEIRA, A.I.T., ALEXANDRE, G.P., MAHMOUD, T.S., "Babaçu (Orbignya sp): Caracterização física de frutos e utilização de solventes orgânicos para extração de óleo", Biochemistry and Biotechnology Reports, v. 2, n. 3esp, pp. 126-129, 2013.

[26] MACHADO, G.C., CHAVES, J.B.P., ANTONIASSI, R., "Physical and chemical characterization and fatty acid composition of babassu oil", Ceres, v. 53, n. 308, pp. 463-470, 2006. 
[27] DESCHAMPS, C., MONTEIRO, R., MACHADO, M. P., et al., "Evaluation of Mentha arvensis, Mentha x piperita and Mentha spp. genotypes for menthol production", Horticultura Brasileira, v. 31, n. 2, pp. 178-183, 2013.

[28] GUNSTONE, F.D., SHAHIDI, F., Bailey's Industrial il \& Fat Products, John Wiley \& Son, 6a ed., v.1, pp. 213-268, New York, 2005.

[29] DESTILARIA BAURU, Disponível em:< http://www.destilariabauru.com.br/>, Acessado em Agosto de 2017.

[30] PUBCHEM., U.S. National Library of Medicine - National Center for Biotechnology Information, Disponível em: 〈http://pubchem.ncbi.nlm.nih.gov>, Acessado em Agosto de 2017.

[31] POORQASEMI, E., ABOOTALEBI, O., PEIKARI, M., et al., "Investigating accuracy of the Tafel extrapolation method in $\mathrm{HCl}$ solutions”, Corrosion Science, v. 51, n. 5, pp. 1043-1054, 2009.

[32] TORRES, V.V., CABRAL, G.B., SILVA, A.C.G.D., et al., "Inhibitory action of papaya seed extracts on the corrosion of carbon steel in 1 mol.L $\mathrm{L}^{-1} \mathrm{HCl}$ solution", Química Nova, v. 39, n. 4, pp. 423-430, 2016.

[33] ALCONCHEL-GAGO, F., ANTAMARÍA, A., TÚNEZ, I., "Antioxidant effect of oleic acid and hydroxytyrosol in an experimental model similar to Huntington's disease”, Actual Med, v. 99, pp. 60-4, 2014.

[34] GENTIL, V., Corrosão, Editora LCT, $6^{\circ}$ edição, Rio de janeiro, 2011.

[35] VASZILCSIN, C.G., DAN, M.L., ENACHE, A.F., et al., "Inhibiting Corrosion Process of Carbon Steel in Sodium Chloride Aqueous Solution by Capsaicin Extract”, Series of Chemistry and Environmental Engineering, v. 61 (75), 1, 2016.

[36] PRADITYANA, A., SHAHAB, A., NOEROCHIM, L., et al. "Inhibition of Corrosion of Carbon Steel in 3.5\% NaCl Solution by Myrmecodia Pendans Extract”, International Journal of Corrosion, v. 2016, 2016.

[37] BOZORG, M., SHAHRABI, F.T., NESHATI, J., et al., "Myrtus communis as green inhibitor of copper corrosion in sulfuric acid", Industrial \& Engineering Chemistry Research, v. 53, n. 11, pp. 4295-4303, 2014.

[38] LI, X., DENG, S., FU, H., et al., "Inhibition effect of 6-benzylaminopurine on the corrosion of cold rolled steel in H2SO4 solution”, Corrosion Science, v. 51, n. 3, pp. 620-634, 2009.

[39] GERENGI, H., DAROWICKI, K., BEREKET, G., et al., "Evaluation of corrosion inhibition of brass118 in artificial seawater by benzotriazole using Dynamic EIS", Corrosion Science, v. 51, n. 11, pp. 2573 2579, 2009.

\section{ORCID}

Jhonatan Peres de Sousa

Renato Conde dos Santos

Camilla Bezerra Ferreira de Souza

Rita de Cássia Silva Costa Neta

Gedeon Silva Reis

Maria Eliziane Pires de Souza

Carmem Célia Francisco do nascimento https://orcid.org/0000-0003-3047-6870

https://orcid.org/0000-0002-1350-1133

https://orcid.org/0000-0003-0954-0620

https://orcid.org/0000-0002-2756-491X

https://orcid.org/0000-0002-2117-2990

https://orcid.org/0000-0002-9184-3921

https://orcid.org/0000-0002-3918-0366 